\title{
The Hubble Constant and Its Relationship to the Dirac Large Numbers Problem
}

\author{
Dapeng Qian* \\ Public Security Bureau of Yingkou, Liaoning Province, China \\ Email: qdpnew@sina.com
}

\begin{abstract}
Based on an improved special relativity that accommodates to the uncertainty principle, a theoretical relationship between the Hubble constant and several fundamental constants has been derived, resulting in the calculated value of $H_{0}=70.937 \mathrm{~km}^{\cdot} \mathrm{s}^{-1} \cdot \mathrm{Mpc}^{-1}$, which compares extremely well with current measured results. The new equation and underlying theory also shed new light into the mystery of the Dirac large numbers problem. Finally, this paper mentions also an important experiment to test the improved special relativity.
\end{abstract}

Keywords: Hubble constant; Dirac large numbers; Improved special relativity.

\section{A Brief Review of History}

After the Hubble law was discovered, many researchers soon noticed that there were a series of dimensionless large numbers that were more or less proportional to the integer powers of $10^{19}$, such as the ratio of the electric to the gravitational force between proton and electron, $\mathrm{N}_{1} \approx\left(10^{19}\right)^{2}$, and the ratio of the universe Hubble radius to the classical radius of electron, $\mathrm{N}_{2} \approx\left(10^{19}\right)^{2}$. The results may be expressed by a "mysterious numbers relation":

$$
\begin{aligned}
& \sqrt[3]{\frac{\hbar^{2} H_{0}}{c G}} \approx m_{\pi} \\
& H_{0} \approx \frac{c G m_{\pi}^{3}}{\hbar^{2}}
\end{aligned}
$$

where, $H_{0}$ denotes the Hubble constant, $c$ denotes the speed of light, $G$ denotes the gravitational constant, $\hbar$ denotes the Plank constant, $m_{\pi}$ denotes the pion mass. Dirac speculated that the correlations between large numbers are not meaningless coincidences, but hint a "fundamental as yet unexplained truth". Based on such a premise, he proposed the famous large numbers principle and speculated that the gravitational constant $G$ should decrease with time ${ }^{[1-2]}$.

Although Dirac's fascination with the large numbers problem was eye-opening his research program was less successful. As we can see, formula (1) is merely a numerical relationship expressed in dimensions and orders of magnitude, which has neither clear conceptual definition nor rigorous logical foundation; the predicted decreasing rate of $G$ had not been supported by astronomical observations; the estimated value of the Hubble constant, $H_{0} \approx 827 \mathrm{~km} \cdot \mathrm{s}^{-1} \cdot \mathrm{Mpc}^{-1}$, deviates greatly from the measured values. Therefore Dirac's theory now attracts little attention from the research community.

Nevertheless, the large numbers relation, as puzzling as it has been, does exist. We should not ignore it just because there were some weaknesses in Dirac's work. Now great progress has been made in observational cosmology, which allows us to reexamine the large number problem and further explore "Is there a correlation between the Hubble constant and other fundamental physical constants? What is the accurate relationship exactly?"

\section{Contributions of the Author's Work}

A new interpretation of the Hubble constant and its relationship to the Dirac large numbers problem has been made possible by the author's work on an improved special relativity ${ }^{[3]}$. The new theory is 
based on a 4-dimensional space-time cylinder model of quarks and leptons that incorporates the time translational symmetry, the 3-dimensional space spherical symmetry and the uncertainty principle of quantum mechanics, thus unifying the special relativity and quantum mechanics in a novel way that is different from the quantum electrodynamics.

Based on the new theory, an accurate relationship is found between the Hubble constant and several other fundamental constants:

$$
H_{0}=\frac{c G m_{e} m_{n}^{2}}{2 \hbar^{2}}
$$

where $m_{e}$ denotes the electron mass, $m_{n}$ denotes the neutron mass. Since the values of all fundamental constants on the right-hand side are known, the Hubble constant can be calculated from equation (2) to be

$$
H_{0}=70.937 \mathrm{~km} \cdot \mathrm{s}^{-1} \cdot \mathrm{Mpc}^{-1}
$$

The formula (2) as a result from a fundamental physical theory does not involve any astronomical observation amounts (such as the red-shift, the apparent magnitude, etc.) in its derivation process, so it is interesting to compare the theoretical value (3) with the measured values of $H_{0}$. Table 1 lists recently published measured values (their mean value is $H_{0}=70.73 \mathrm{~km} \cdot \mathrm{s}^{-1} \cdot \mathrm{Mpc}^{-1}$ ). Also, it is worth mentioning that there was an "old" measured value from the ten-year observation results of the Hubble space telescope key project ${ }^{[9]}, H_{0}=72(71) \pm 4 \pm 7 \mathrm{~km} \cdot \mathrm{s}^{-1} \cdot \mathrm{Mpc}^{-1}$. We can see that the theoretical value (3) is consistent with these measured values.

Table 1. Recent measured values of the Hubble constant

\begin{tabular}{cccccc}
\hline Project & MCP $^{[4]}$ & CHP $^{[5]}$ & WMAP $^{[6]}$ & Planck $^{[7]}$ & SH0ES $^{[8]}$ \\
\hline Publication & 2012 & 2012 & 2014 & 2015 & 2016 \\
$H_{0}\left(\mathrm{~km}^{-1} \mathrm{~s}^{-1} \cdot \mathrm{Mpc}^{-1}\right)$ & $68.9 \pm 7.1$ & $74.3 \pm 2.1$ & $69.6 \pm 0.7$ & $67.8 \pm 0.9$ & $73.03 \pm 1.79$ \\
\hline
\end{tabular}

A direct comparison of equations (2) and (1) reveals significantly new insights into the origins of Dirac large numbers. Both equations have similar constructions and contain several common fundamental constants $c, G, \hbar, H_{0}$. However, there is a key difference. While equation (1) is merely an empirical formula, equation (2) is strictly mathematical and derived from a conceptually rigorous physical theory that incorporates both the special relativity and the uncertainty principle of quantum mechanics. In light of equation (2) and the new theory, there is indeed a specific correlation between the Hubble constant $H_{0}$ and fundamental physical constants $c, G, \hbar$, as well as the masses of two special particles $m_{e}$ and $m_{n}$ (but not the pion mass $m_{\pi}$ !). The above-mentioned large numbers $\mathrm{N}_{1}$ and $\mathrm{N}_{2}$ are also elucidated by equation (2). Therefore, now we can say that the "fundamental truths" hidden in the mysterious large numbers have been disclosed by the new equation and underlying theory.

In addition to the Hubble constant and the large numbers problem, the improved special relativity also has many other important applications. For example, the new theory predicts the existence of the Lorentz invariance violation (LIV) and derives an exact formula to directly calculate the LIV coefficient $\xi$, For UHECR protons above $4 \times 10^{19} \mathrm{eV}$, it can be calculated that $|\xi|<4.5 \times 10^{-30}<<10^{-23}$, so too weak to change the GZK cutoff, which is consistent with the observations by HiRes ${ }^{[10]}$ and Auger ${ }^{[1]}$. Most critically, the new theory predicts an unusual effect, which can cause an emission of electrons of unusually high energy, much higher than the beam energy, in the downstream direction of the electron storage ring RF cavity. The detection of such small probability events constitutes a crucial proof or disproof of the new theory. Some preliminary experiments have indeed shown some positive results ${ }^{[12]}$.

\section{References}

1. P. A. M. Dirac, The cosmological constants, Nature, 139, 323, (1937)

2. S. Weinberg, Gravitation and Cosmology: Principles and Applications of the General Theory of Relativity, John Wiley \& Sons, Inc. 619-621, (1972) 
3. Dapeng Qian, A New Version of Special Relativity Absorbed the Uncertainty Principle: Its Content as Well as Application and Experimental Test, Journal of Modern Physics, 5, 1146-1166, (2014) [http://dx.doi.org/10.4236/jmp.2014.512117]

4. M. J. Reid, et al, The Megamaser Cosmology Project: IV. A Direct Measurement of the Hubble Constant from UGC 3789, (2012) [arXiv:1207.7292]

5. W. L. Freedman, et al, Carnegie Hubble Program: A Mid-Infrared Calibration of the Hubble Constant, Astrophys. J. 758:24 (2012) [arXiv:1208.3281]

6. C. L. Bennett, et al, The 1\% Concordance Hubble Constant, (2014) [arXiv:1406.1718]

7. Planck Collaboration, Planck 2015 results. XIII. Cosmological parameters, [arXiv:1502.01589] (2015)

8. Adam G. Riess, et al, A $2.4 \%$ Determination of the Local Value of the Hubble Constant, (2016) [arXiv:1604.01424]

9. W. L. Freedman, et al, Final Results from the Hubble Space Telescope Key Project to Measure the Hubble Constant, Astrophys.J.553:47-72, (2001) [arXiv:astro-ph/0012376v1]

10. HiRes Collaboration, First Observation of the Greisen-Zatsepin-Kuzmin Suppression, Phys. Rev. Lett. 100, 101101 (2008) [arXiv:astro-ph/0703099]

11. Pierre Auger Collaboration, Recent results from the Pierre Auger Observatory, (2015) [arXiv:1503.09173]

12. Dapeng Qian, Suspicious Signs of Emission of $38 \mathrm{GeV}$ Electrons Have Been Detected in the $0.8 \mathrm{GeV}$ Electron Storage Ring, Journal of Liaoning University (Natural Science Edition) 37, 236-240, (2010) 